\title{
latrogenic neurological injury after radiofrequency ablation and epidural steroid injections: illustrative cases
}

\author{
Lisa B. E. Shields, MD, ${ }^{1}$ Vasudeva G. Iyer, MD, ${ }^{2}$ Yi Ping Zhang, MD, ${ }^{1}$ and Christopher B. Shields, $M D^{1,3}$ \\ ${ }^{1}$ Norton Neuroscience Institute, Norton Healthcare, Louisville, Kentucky; ${ }^{2}$ Neurodiagnostic Center of Louisville, Louisville, Kentucky; and ${ }^{3}$ Department of Neurological Surgery, \\ University of Louisville School of Medicine, Louisville, Kentucky
}

\begin{abstract}
BACKGROUND Neck pain is often chronic and disabling. Cervical facet joint injections and epidural steroid injections are frequently used to manage chronic neck pain and cervicogenic headaches. While minimal side effects are commonly associated with these treatments, severe complications are exceedingly rare.

OBSERVATIONS The authors report 4 cases of iatrogenic neurological injury after radiofrequency ablation (RFA) and epidural steroid injections. One patient experienced left shoulder, scapular, and arm pain with left arm and hand weakness that developed immediately after RFA for chronic neck pain. Electromyography/nerve conduction velocity (EMG/NCV) studies confirmed denervation changes in the left C8-T1 distribution. Three patients complained of numbness and weakness of the hands immediately after an interlaminar cervical epidural block. One of these patients underwent EMG/ NCV that confirmed denervation changes occurring in the left C8-T1 distribution.

LESSONS Spine surgeons and pain management specialists should be aware of neurological injuries that may occur after cervical RFA and epidural steroid injections, especially after a multilevel cervical procedure and with severe cervical spinal stenosis. EMG/NCV studies plays an important role in detecting and localizing neurological injury and in differentiating from conditions that mimic cervical root injuries, including brachial plexus trauma due to positioning and Parsonage-Turner syndrome.

https://thejns.org/doi/abs/10.3171/CASE2148
\end{abstract}

KEYWORDS neurosurgery; radiofrequency ablation; epidural steroid injections; cervical; electromyography; nerve conduction study

Approximately $10 \%-20 \%$ of adults experience neck pain at any given time that is associated with a high frequency of recurrence. ${ }^{1,2}$ In addition, $20 \%-70 \%$ of adults report having neck pain that interferes with their daily activities during their lifetimes. ${ }^{1,2}$ Cervical facet joint injections, such as intra-articular facet joint injections, medial branch blocks, or medial branch radiofrequency ablation (RFA)/radiofrequency neurotomy (RFN), are minimally invasive procedures used to manage chronic neck pain and cervicogenic headaches. ${ }^{1,3-8}$ In cervical RFA, the articular limbs of the dorsal ramus medial branches are ablated, which eliminates the afferent nociceptive input from the adjacent facet joint. ${ }^{9}$ Most patients who undergo RFN are pain free at 6 months, while one-third of patients are pain free at 1 year. ${ }^{4,8,10,11}$ Pain often recurs due to axonal regeneration, necessitating repeat RFN. ${ }^{9,12}$
While major complications are extraordinarily rare after cervical facet joint injections, minor and temporary side effects are more frequent and are related to needle placement, temperature, or drug administration. ${ }^{1,3,4,7,12-15}$ Complications may include infection (epidural abscess or bacterial meningitis), dural puncture, spinal cord or neural trauma, or cerebral infarction secondary to vascular injury to the vertebral artery. ${ }^{1,7,9}$ In Manchikanti and colleagues' study of 3370 cervical facet joint nerve blocks, the most frequent side effect was local bleeding with no major complications. ${ }^{16}$ Other side effects included intravascular penetration in the cervical region in $20 \%$, as well as temporary numbness, dysesthesia, pruritus, and ataxia. $3,7,8,12,14,17$

Despite the approximately 9 million cervical epidural steroid injections performed annually in the United States, they are not ap-

ABBREVIATIONS ACDF = anterior cervical discectomy and fusion; $\mathrm{ADM}=$ abductor digit minimi; $\mathrm{AP}=$ anteroposterior; $\mathrm{APB}=$ abductor pollicis brevis; $\mathrm{BMI}=$ body mass index; $\mathrm{CSF}=$ cerebrospinal fluid; $\mathrm{CT}=$ computed tomography; El = extensor indicis; $\mathrm{EMG}=$ electromyography; FDI $=$ first dorsal interosseous; FPL = flexor pollicis longus; MRI = magnetic resonance imaging; NCV = nerve conduction velocity; RFA = radiofrequency ablation; RFN = radiofrequency neurotomy.

INCLUDE WHEN CITING Published May 10, 2021; DOI: 10.3171/CASE2148.

SUBMITTED January 22, 2021. ACCEPTED February 8, 2021.

(C) 2021 The authors, CC BY-NC-ND 4.0 (http://creativecommons.org/licenses/by-nc-nd/4.0/). 
proved by the US Food and Drug Administration, and no randomized controlled trials have documented their safety and efficacy. ${ }^{18}$ A host of severe complications are associated with cervical epidural steroid injections, including epidural hematomas, infection (abscess/meningitis), and neurological deficits due to intramedullary (quadriparesis/quadriplegia) and intravascular injections (vertebral artery injections leading to spinal cord, brainstem, and cerebellar strokes). ${ }^{18-20}$ Cerebrovascular accidents may follow cervical epidural steroid injections due to particulate steroid matter in the methylprednisolone injection solution that embolized to the distal arterial branches. ${ }^{18,21}$

We present 4 exceedingly rare cases of patients who experienced a neurological injury after RFA and epidural steroid injections. The method of injury, differential diagnoses, treatment, and prognosis are discussed. The narrowest anteroposterior (AP) diameter of cervical spinal stenosis was also determined for cases $2-4$.

\section{Illustrative Cases}

\section{Case 1}

History and Radiological Imaging

A 63-year-old man (body mass index [BMI] $28.48 \mathrm{~kg} / \mathrm{m}^{2}$ ) underwent RFA from $\mathrm{C} 2$ to $\mathrm{C} 5$ on the right for neck pain. The patient stated that he woke up during the procedure and tried to raise his head. Additional sedative was given, and the procedure continued. On awakening from anesthesia, he noted sharp left shoulder and scapular pain radiating down the posterior aspect of the left upper arm to the elbow as well as a tingling sensation radiating down the entire left arm to all fingers. The patient reported left arm and hand weakness. He denied any balance difficulties, gait instability, saddle anesthesia, or bladder/bowel incontinence or retention. The patient was administered methylprednisolone $4 \mathrm{mg}$ (Medrol dose pack, Zoetis) and gabapentin $300 \mathrm{mg} 2$ tablets per day. His past medical history included hyperlipidemia, continuous positive airway pressure dependence, cigarette smoking, and a transient ischemic attack 4 years earlier that affected his speech. His past surgical history recorded an anterior cervical vertebrectomy of $\mathrm{C} 5$ and $\mathrm{C} 6$ followed by cage placement from $\mathrm{C} 4$ to $\mathrm{C} 7$.

Cervical magnetic resonance imaging (MRI) without gadolinium performed 4 days after the RFA revealed no acute changes. The anterior cervical spine fusion from $\mathrm{C} 4$ to $\mathrm{C} 7$ was observed with chronic rightsided myelomalacia at the superior $\mathrm{C} 5$ vertebral body level. Moderate spinal canal stenosis and moderate neural foraminal stenosis at C3-4 as well as severe neural foraminal stenosis at C4-5 on the left and at C6-7 bilaterally were noted.

\section{Physical Examination}

Decreased range of motion of the neck with tenderness to palpation of the left shoulder was detected. Marked weakness of the left abductor pollicis brevis (APB), left flexor pollicis longus (FPL), first dorsal interosseous (FDI), abductor digit minimi (ADM), and extensor pollicis longus was noted. There was decreased sensation to pinprick over the left thumb and index finger.

\section{Electromyography/Nerve Conduction Velocity of the Arms}

Electromyography (EMG) performed 6 weeks after the onset of symptoms demonstrated fibrillations and positive sharp waves in the left APB, FDI, and ADM. The patient was able to recruit only a few motor units in these muscles. The extensor indicis (EI), the $\mathrm{FPL}$, and, to a lesser extent, the triceps also showed a decrease in

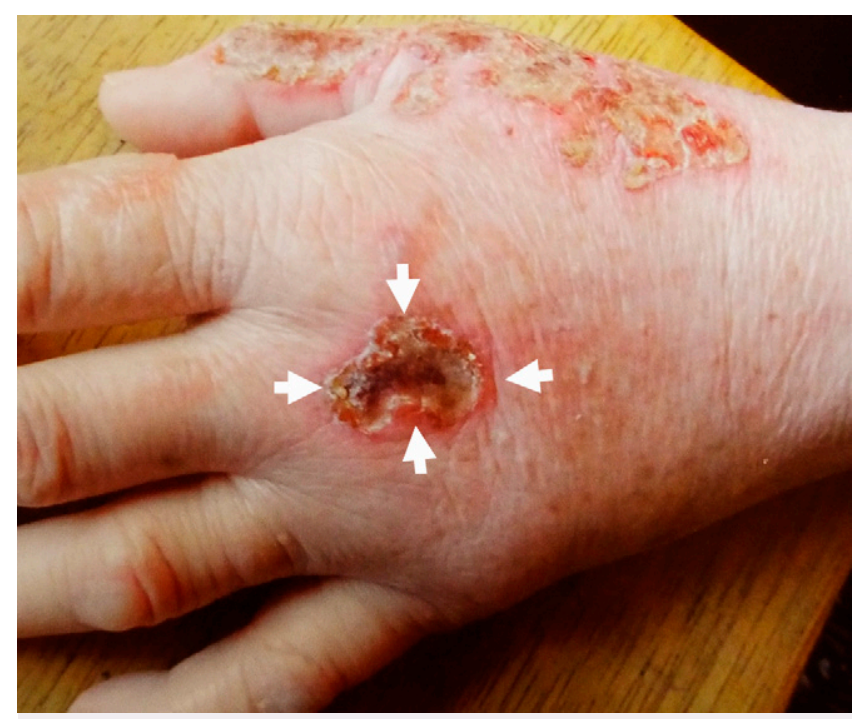

FIG. 1. Photograph of the dorsum of the left hand taken 2 hours after the injection, demonstrating severe ulceration (arrows) as well as excoriation over the dorsum of the thumb and proximal radial aspect of the hand.

motor units with only 1-2 motor units. These findings confirmed denervation changes in the left C8-T1 radicular distribution.

\section{Follow-Up}

The patient's left arm pain persisted after the RFA. He underwent a second RFA from C2 to C5 on the left 8 months later. The patient continued to experience neck and left arm pain and left arm weakness 7 months later. He was taking gabapentin $300 \mathrm{mg} 6$ tablets daily and meloxicam $15 \mathrm{mg}$ daily. He was unable to work or exercise. A cervical myelogram and post-myelographic computed tomography (CT) scan demonstrated a large calcified ligament/ osteophyte complex with a Charcot joint at the $\mathrm{T} 1$ spinous process.

\section{Case 2}

History and Radiological Imaging

A 71-year-old woman (BMI $24.46 \mathrm{~kg} / \mathrm{m}^{2}$ ) underwent a midline interlaminar epidural steroid injection at $\mathrm{C} 7-\mathrm{T} 1$ for neck and arm pain. A 20-gauge 3.5-inch Touhy needle was advanced until loss of resistance with a fluid-filled pulsator syringe was noted. Omnipaque 240 (2 ml; GE Healthcare) was injected at C7-T1, which showed a linear filling pattern in the midline posteriorly, suggesting intramedullary contrast injection. The patient complained of immediate cervical discomfort. The needle was removed and then reinserted at C7-T1 in the midline and advanced until loss of resistance was noted. Two milliliters of contrast agent was injected, which showed epidural spread. Two milliliters of preservative-free saline and $40 \mathrm{mg}$ of Depo Medrol (Pfizer) were then administered into the epidural space.

Immediately after the procedure, the patient complained of increased neck and left shoulder pain as well as weakness of left hand grip. She described allodynia when hot or cold water was applied to her left arm. Blisters developed on the dorsal aspect of her left hand within 2 hours of the injection (Fig. 1). The patient 

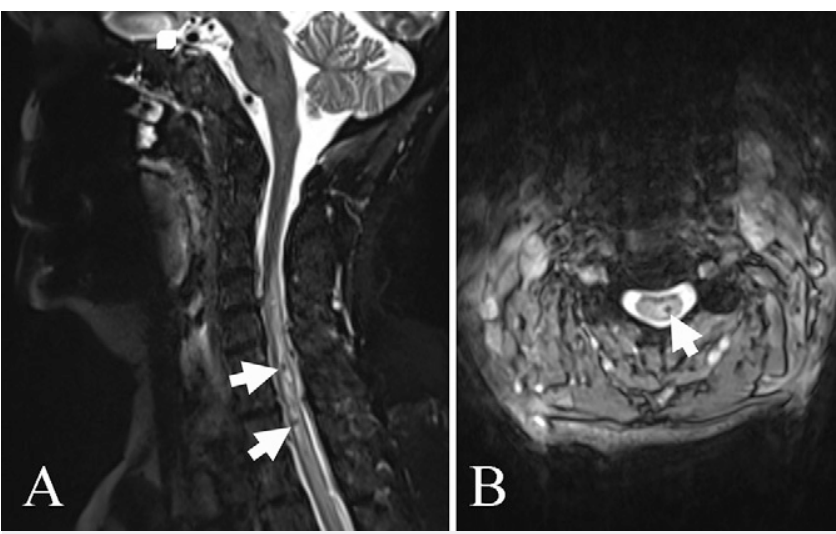

FIG. 2. A: Sagittal T2 MRI (short tau inversion recovery) showing intramedullary blood (arrows) and edema. B: Axial view showing a dark area of blood (arrow) and surrounding edema at C5-6.

was prescribed a Medrol dose pack. Her past medical history was significant for hypertension, fibromyalgia, hyperthyroidism, myocardial infarction, and coronary artery disease. She had undergone anterior cervical discectomy and fusion (ACDF) of C6-7 30 years earlier.

Cervical MRI with and without gadolinium contrast performed 1 day after the epidural steroid injection revealed increased T2 signal within the cervical spinal cord, extending from C3 into the thoracic spine (Fig. 2). There was also hemosiderin deposition in the cervical spinal cord from $\mathrm{C} 4$ to $\mathrm{C} 7$. The diameter from the posterior aspect of the vertebral body to the inner aspect of the laminar arch at C5-6 was $8 \mathrm{~mm}(12-16 \mathrm{~mm})$.

\section{Physical Examination}

Profound weakness of the left FDI, ADM, APB, FPL, flexor digitorum profundus, and El was detected (Fig. 3). There was decreased pinprick sensation of all digits and the forearm on the left. The triceps and biceps deep tendon reflexes were symmetrical.

\section{EMG/Nerve Conduction Velocity of the Arms}

EMG performed 6 months after the onset of symptoms showed fibrillations and positive waves in the left FDI with no motor units. The APB demonstrated fibrillations with a single motor unit. The El, FPL, and flexor carpi ulnaris revealed a significant decrease in motor units. These findings confirmed denervation changes in the left C8-T1 distribution. The normal sensory potentials in the ulnar nerve distribution suggested a preganglionic injury at the C8-T1 root level. No reinnervation changes were noted in the left intrinsic hand muscles.

\section{Case 3}

History and Radiological Imaging

A 62-year-old woman (BMI $33.67 \mathrm{~kg} / \mathrm{m}^{2}$ ) underwent an interlaminar epidural steroid injection at C7-T1 for neck pain. A Tuohy needle was advanced to the interlaminar space and into the epidural space under fluoroscopic guidance. The position was verified with a loss-of-resistance technique to air. After confirming the location of the needle, aspiration was negative for blood and cerebrospinal fluid (CSF), and 0.5 ml of Omnipaque NDC 0407-1412-30 (GE Healthcare) was injected. After observing the appropriate epidurogram

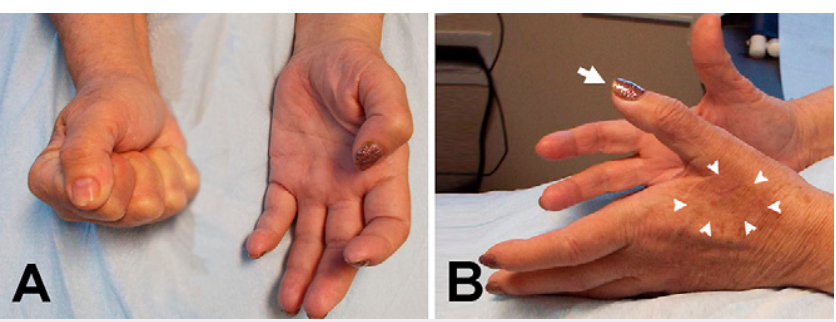

FIG. 3. A: Photograph demonstrating weakness in left hand grip with intact flexion of the distal interphalangeal joint. B: Photograph demonstrating marked atrophy of the FDI muscle (arrowheads) with failure of complete extension of the dorsal interphalangeal joint (arrow).

with lateral and posteroanterior views, $1 \mathrm{ml}$ of a 3-ml solution reconstituted from $10 \mathrm{mg}$ of dexamethasone was injected. The patient immediately elevated both shoulders and hyperextended the head. The injection was promptly aborted, and within a few minutes, an additional $0.5 \mathrm{ml}$ of contrast was injected. While aspiration was negative, a radiograph revealed contrast spread posterior to the dura.

The patient immediately noted bilateral hand numbness and tingling with decreased right grip strength as well as onset of pain of the left posterior shoulder and abdominal "hardness." Her blood pressure was elevated, and her pulse and oxygen saturation were normal. Fentanyl and labetalol were administered. As the patient's symptoms did not respond to treatment, she was hospitalized for 3 days.

A head CT demonstrated pneumocephalus with multifocal locules of gas in the subdural space at the tentorium and the middle cranial fossa bilaterally. Cervical MRI with and without gadolinium revealed intramedullary signal from $\mathrm{C} 3$ to $\mathrm{T} 7$ with gas manifesting as low T1 and T2 signal with surrounding focal fluid in the cord at C5 (Fig. 4). There was increased signal extending above this level of gas by approximately $1 \mathrm{~cm}$ to the $\mathrm{C} 4-5$ level. Numerous bubbles of gas were identified within the subarachnoid space. The diameter from the posterior aspect of the vertebral body to the inner aspect of the laminar arch at C6-7 was $6 \mathrm{~mm}(12-16 \mathrm{~mm})$.

Physical Examination

The patient's strength was $2 / 5$ in the distal upper extremities bilaterally and $4 / 5$ proximally. Her sensation to light touch was intact, and her deep tendon reflexes were $2+$ and symmetric.

\section{Follow-Up}

Within 8 days of the cervical epidural injection, the numbness of the upper extremities had resolved; however, she continued to experience right hand weakness.

\section{Case 4}

History and Radiological Imaging

A 63-year-old woman (BMl $43.38 \mathrm{~kg} / \mathrm{m}^{2}$ ) underwent an interlaminar epidural steroid injection at C6-7 for neck pain. A 20-gauge 3.5-inch Tuohy needle was used. The epidural space was identified using the loss-of-resistance technique to air. Aspirations were negative for blood or CSF. As soon as there was loss of resistance, the patient moved, at which time paresthesias were noted in the arms and trunk. The needle was immediately removed, and no steroid, saline, or contrast was injected. 


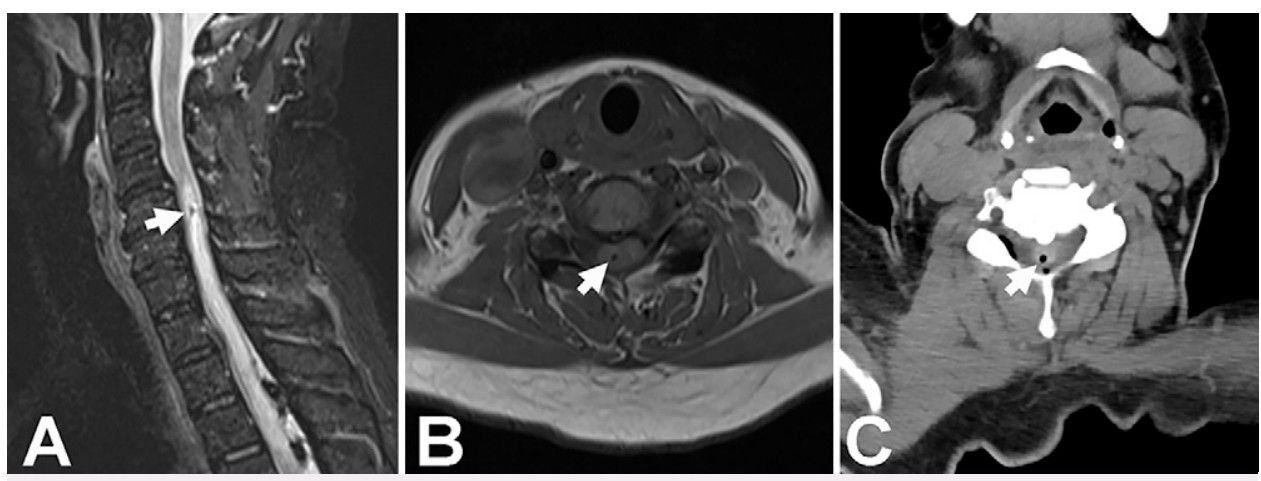

FIG. 4. A: Sagittal T2-weighted MRI scan demonstrating air in the spinal cord at C5-6 (arrow) and spinal cord edema caudal to that area. Three dark areas in the inferior aspect of the MRI scan represent air in the subdural space. Axial views of the MRI (B) and CT (C) scans showing intramedullary air (arrows).

After the procedure, the patient was "agitated" and unable to follow any commands. She complained of neck pain, numbness of the right side of the body (trunk, abdomen, arm, and leg), and weakness of the right arm and leg. She was given gabapentin 200 mg twice per day. She had a history of hypertension, and she had undergone an ACDF at C4-5.

Cervical MRI with and without gadolinium contrast demonstrated two focal areas of abnormal signal within the spinal cord at C3 (8.0 $\mathrm{mm}$ in length and $4.0 \mathrm{~mm}$ in AP and transverse dimensions) and $\mathrm{C} 4(8.0 \mathrm{~mm}$ in length and $5.0 \mathrm{~mm}$ in $\mathrm{AP}$ and transverse dimensions) with decreased signal on T1 and T2 sequences (Fig. 5). These findings suggested acute blood in the spinal cord, and the T2 MRI sequence showed minimal surrounding edema. The diameter from the posterior aspect of the vertebral body to the inner aspect of the laminar arch at C4-5 was $6.2 \mathrm{~mm}(12-16 \mathrm{~mm})$.

\section{Physical Examination}

The patient experienced tingling of the right upper extremity, with dysesthesia extending from the right $\mathrm{C} 6$ to the T10-11 dermatome. Fine touch in the right arm, leg, and trunk was intact. The patient had 4/5 strength in the deltoid, triceps, and brachioradialis as well as diminished right hand grip. The following deep tendon reflexes were absent: right triceps, biceps, and brachioradialis; left biceps and brachioradialis; and Achilles bilaterally. The patellar deep tendon reflexes were 2/4 bilaterally, and the left triceps deep tendon reflex was $1 / 4$

Follow-Up

The patient underwent a 3-week course of physical therapy. Three months after the cervical epidural injection, she had persistent numbness and tingling of the right middle, index, and little fingers. Her right arm remained weaker than her left arm. Her right thoracic area numbness had improved. Cervical MRI 3 months after the cervical epidural injection revealed no residual hemorrhage or edema.

\section{Discussion}

Neurological complications associated with RFA have rarely been reported in the literature. A single case of L5 root injury, kyphosis/dropped head syndrome after multilevel cervical lesioning, transient tetraplegia, hoarseness, spinal accessory nerve injury, and Horner's syndrome has been documented. ${ }^{9,17,22-26}$ Ahmed and colleagues presented a patient who underwent multilevel cervical radiofrequency facet neurotomies for chronic headaches. ${ }^{17}$ Immediately after the procedure, the patient's chin assumed a flexed position on his chest, and he was unable to extend, rotate, or laterally flex his neck. EMG performed 8 months later revealed active denervation of
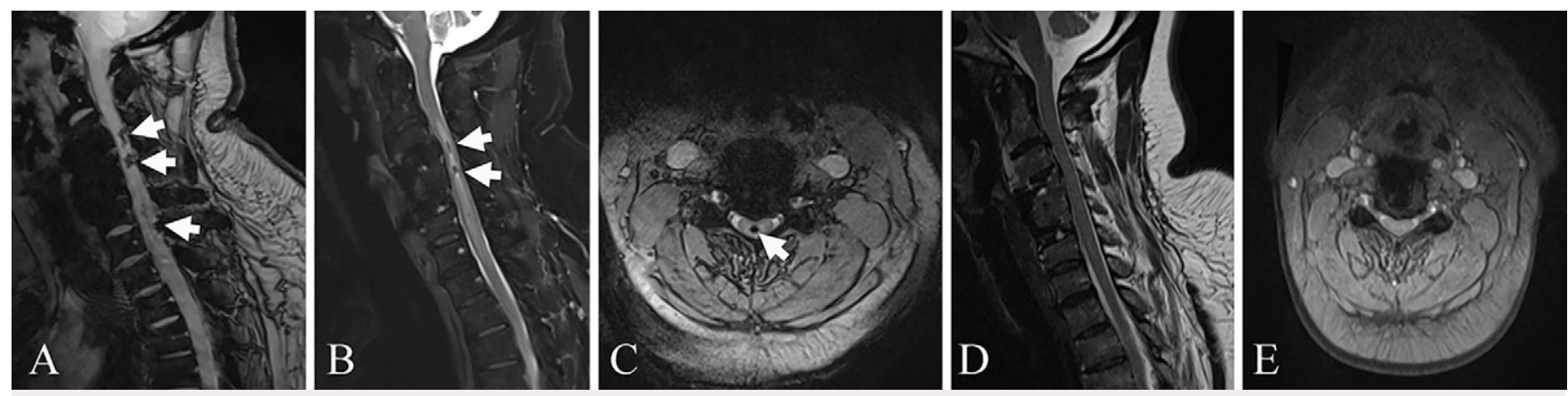

FIG. 5. A: Sagittal T2 MRI (gradient echo [GRE]) scan showing blood (arrows) within the spinal cord at C3-4, C4-5, and C6-7. B: Sagittal T2 with fat suppression shows air and hemorrhage (arrows) with extensive intramedullary edema. C: Axial T2 (GRE) MRI scan demonstrating hemorrhage and gas bubble (arrow) at C3-4. Sagittal (T2; D) and axial (GRE; E) MRI scans show resolution of hemorrhage, air, and edema 3 months after the injection; however, underlying cervical spinal stenosis is evident. 
the cervical paraspinous musculature with no evidence of motor neuron function. Despite narcotic medications, gabapentin, and intrathecal baclofen, the patient continued to experience severe neck pain. He underwent a three-level anterior cervical discectomy with placement of intervertebral allograft followed by a C4 laminectomy and C3-4 and C4-5 bilateral foraminotomies with a posterior cervical fusion from $\mathrm{C} 3$ to $\mathrm{C} 6$. The neck pain persisted postoperatively, but the chin-on-chest deformity was corrected. These authors proposed that the kyphotic deformity was attributable to denervation of the dorsal primary rami at multiple cervical levels secondary to RFN. ${ }^{17}$ They suggested minimizing the number of cervical levels treated simultaneously and to confirm radiographically that the lesioning needle was accurately placed.

Stoker and colleagues reported a patient who underwent bilateral RFA of the C2-4 medial articular branch and third occipital nerve for neck pain and headaches. ${ }^{9}$ The patient experienced neck pain, cervical extensor weakness, and occipital hyperesthesia immediately following the procedure. Within 5 months, she demonstrated a chin-on-chest dropped head deformity and an inability to extend the neck. The patient underwent a laminectomy from C4 to $\mathrm{C} 7$ and an instrumented posterior instrumented fusion from $\mathrm{C} 2$ to T2. Six months postoperatively, the cervical alignment had improved, and the neck pain had resolved. These authors suggested that the etiology of the cervical extensor muscle denervation was caused by RFA of multiple dorsal rami motor components. ${ }^{9}$ They also cautioned against performing multilevel cervical RFA. Similarly, McDonald and colleagues advised against single-stage bilateral or multilevel cervical RFA because excessive denervation may promote neuromuscular instability and secondary deformity. ${ }^{11}$

Persistent or recurrent neck pain commonly follows cervical spine surgery. Approximately $15 \%-40 \%$ of patients experience disabling neck pain caused by perineural and epidural scarring with nerve root adhesions and muscle spasm. ${ }^{6}$ In Klessinger's study of 242 patients who underwent cervical spine surgery, 31\% had persistent neck pain due to zygapophysial joint pain postoperatively. ${ }^{6}$ These authors reported that more patients experienced neck pain after a two-level operation than during a single-level operation. Their findings suggest that the extent of previous surgery and number of levels treated are major risk factors for postoperative neck pain. Because the patients in our series (cases 1, 2, and 4) had undergone extensive spine surgery, it is not unexpected that neck pain occurred, necessitating RFA and cervical epidural injections.

The differential diagnosis of the cause of cervical nerve root injury after RFA includes a brachial plexus injury due to shoulder and neck positioning. Intraoperatively, the patient's shoulders are retracted downward when in the prone position. This maneuver allows visualization of the lower vertebral levels on lateral cervical spine radiography performed for correct localization. If the shoulders are forcefully pulled down, the superior trunk of the brachial plexus may be stretched and injured, resulting in similar symptoms as those in a cervical root injury. Parsonage-Turner syndrome (idiopathic brachial plexopathy or neuralgic amyotrophy) is a rare condition composed of numerous symptoms such as shoulder pain, motor weakness, dysesthesias, and numbness of the upper extremity. ${ }^{27}$ Although the etiology is unknown, this disorder may occur postoperatively and may be postinfectious, posttraumatic, or postvaccination. Sensory and motor components may occur in Parsonage-Turner syndrome and may mimic cervical nerve root injury. EMG/nerve conduction velocity (NCV) studies are valuable in differentiating between these conditions.

\section{Observations}

Case 1 is the first in the literature to describe a cervical nerve root injury after RFA. While the RFA was performed from C2 to C5 on the right, the patient's symptoms after the procedure were on the left. We speculate that the trajectory of the ablation may have changed when the patient awoke, tried to move his head, and was quickly sedated. The multilevel RFA presumably increased the risk of complications. Case 2 combined several mechanisms that led to the patient's symptoms after cervical epidural spinal injections. It is likely that the injection of contrast into the dorsal column of the spinal cord as well as the C8-T1 nerve roots may have caused the numbness and the muscle weakness. The rash on the left hand may have been caused by autonomic dysfunction at C8-T1. In case 3, the patient's symptoms arose after cervical epidural spinal injections with the needle being inadvertently placed into the dorsal column of the spinal cord at $\mathrm{C} 5$. This resulted in the cephalad migration of gas observed on the head CT as well as the numbness, tingling, and weakness of the hands bilaterally by affecting the C8-T1 nerve roots. In case 4 , the symptoms presented after a cervical epidural injection. It is most likely that the needle hit the spinal cord, causing injury and hemorrhage within the spinal cord. A unique aspect of the present case series was the determination that preexisting cervical spinal stenosis has an increased risk of neurological complications after epidural injections for pain control.

\section{Lessons}

Spine surgeons and pain management specialists should be cognizant of neurological injuries that may occur after RFA and cervical epidural injections, especially with a multilevel ( $>2$ levels) cervical procedure and severe cervical spinal stenosis. EMG/NCV play an important role in precise localization of cervical root lesions and in differentiating between conditions that mimic cervical nerve root injuries, such as brachial plexus injuries due to shoulder and neck positioning as well as Parsonage-Turner syndrome.

\section{Acknowledgments}

We acknowledge Norton Healthcare for their continued support.

\section{References}

1. Falco FJ, Manchikanti L, Datta S, et al. Systematic review of the therapeutic effectiveness of cervical facet joint interventions: an update. Pain Physician. 2012;15(6):E839-E868.

2. Sinnott PL, Dally SK, Trafton J, et al. Trends in diagnosis of painful neck and back conditions, 2002 to 2011. Medicine (Baltimore). 2017;96(20):e6691.

3. Chazen JL, Ebani EJ, Virk M, et al. CT-guided block and radiofrequency ablation of the $\mathrm{C} 2$ dorsal root ganglion for cervicogenic headache. AJNR Am J Neuroradiol. 2019;40(8):1433-1436.

4. Engel A, Rappard G, King W, Kennedy DJ. The effectiveness and risks of fluoroscopically-guided cervical medial branch thermal radiofrequency neurotomy: a systematic review with comprehensive analysis of the published data. Pain Med. 2016;17(4):658-669.

5. Husted DS, Orton D, Schofferman J, Kine G. Effectiveness of repeated radiofrequency neurotomy for cervical facet joint pain. $J$ Spinal Disord Tech. 2008;21(6):406-408.

6 . Klessinger $\mathrm{S}$. Radiofrequency neurotomy for the treatment of therapy-resistant neck pain after ventral cervical operations. Pain Med. 2010;11(10):1504-1510. 
7. Nagar VR, Birthi P, Grider JS, Asopa A. Systematic review of radiofrequency ablation and pulsed radiofrequency for management of cervicogenic headache. Pain Physician. 2015;18(2):109-130.

8. Nguyen T, Chan K, Chryssidis S, Selby M. CT guided radiofrequency ablation of the cervical medial branch using a lateral approach in the supine patient. J Spine Surg. 2017;3(3):463-467.

9. Stoker GE, Buchowski JM, Kelly MP. Dropped head syndrome after multilevel cervical radiofrequency ablation: a case report. J Spinal Disord Tech. 2013;26(8):444-448.

10. MacVicar J, Borowczyk JM, MacVicar AM, et al. Cervical medial branch radiofrequency neurotomy in New Zealand. Pain Med. 2012; 13(5):647-654.

11. McDonald GJ, Lord SM, Bogduk N. Long-term follow-up of patients treated with cervical radiofrequency neurotomy for chronic neck pain. Neurosurgery. 1999;45(1):61-68.

12. Lord SM, Barnsley L, Wallis BJ, et al. Percutaneous radiofrequency neurotomy for chronic cervical zygapophyseal-joint pain. N Engl J Med. 1996;335(23):1721-1726.

13. Burnham T, Conger A, Salazar $F$, et al. The effectiveness of cervical medial branch radiofrequency ablation for chronic facet joint syndrome in patients selected by a practical medial branch block paradigm. Pain Med. 2020;21(10):2071-2076.

14. Govind J, King W, Bailey B, Bogduk N. Radiofrequency neurotomy for the treatment of third occipital headache. J Neurol Neurosurg Psychiatry. 2003;74(1):88-93.

15. Wagner AL. CT fluoroscopic-guided cervical nerve root blocks. AJNR Am J Neuroradiol. 2005;26(1):43-44.

16. Manchikanti L, Malla Y, Wargo BW, et al. Complications of fluoroscopically directed facet joint nerve blocks: a prospective evaluation of 7,500 episodes with 43,000 nerve blocks. Pain Physician. 2012; 15(2):E143-E150.

17. Ahmed MM, Lake WB, Resnick DK. Progressive severe kyphosis as a complication of multilevel cervical percutaneous facet neurotomy: a case report. Spine J. 2012;12(10):e5-e8.

18. Epstein NE. Major risks and complications of cervical epidural steroid injections: an updated review. Surg Neurol Int. 2018;9:86.

19. Candido KD, Knezevic N. Cervical epidural steroid injections for the treatment of cervical spinal (neck) pain. Curr Pain Headache Rep. 2013;17(2):314.

20. Lee JK, Chae KW, Ju Cl, Kim BW. Acute cervical subdural hematoma with quadriparesis after cervical transforaminal epidural block. J Korean Neurosurg Soc. 2015;58(5):483-486.
21. Ziai WC, Ardelt AA, Llinas RH. Brainstem stroke following uncomplicated cervical epidural steroid injection. Arch Neurol. 2006; 63(11):1643-1646.

22. Abbott Z, Smuck M, Haig A, Sagher O. Irreversible spinal nerve injury from dorsal ramus radiofrequency neurotomy: a case report. Arch Phys Med Rehabil. 2007;88(10):1350-1352.

23. Huntoon K, Eltobgy M, Mohyeldin A, Elder JB. Lower extremity paralysis after radiofrequency ablation of vertebral metastases. World Neurosurg. 2020;133:178-184.

24. Sutton T. Hoarseness following cervical facet denervation: a case report. Abstract. J Pain. 2011;12(4 suppl):P17.

25. Thakkar NS, Gray MJ, Ramesh SS, et al. Spinal accessory nerve palsy following cervical cooled radiofrequency ablation: a case report and review of the literature. Interv Pain Manag Rep. 2017;1: 199-205.

26. Topcu-Yilmaz P, Kutluk S, Onder F. Horner syndrome as a rare complication of radiofrequency tonsil ablation. J Pediatr Ophthalmol Strabismus. 2018;55:e14-e15.

27. Feinberg JH, Radecki J. Parsonage-Turner syndrome. HSS J. 2010;6(2):199-205.

\section{Disclosures}

The authors report no conflict of interest concerning the materials or methods used in this study or the findings specified in this paper.

\section{Author Contributions}

Conception and design: CB Shields, LBE Shields, lyer. Acquisition of data: CB Shields, LBE Shields, lyer. Analysis and interpretation of data: CB Shields, LBE Shields, lyer. Drafting the article: LBE Shields. Critically revising the article: CB Shields, LBE Shields, lyer, Zhang. Reviewed submitted version of manuscript: CB Shields, LBE Shields, lyer, Zhang. Approved the final version of the manuscript on behalf of all authors: CB Shields, LBE Shields. Administrative/technical/material support: CB Shields, LBE Shields. Study supervision: CB Shields.

\section{Correspondence}

Christopher B. Shields: Norton Neuroscience Institute, Norton Healthcare, Louisville, KY. cbshields1@gmail.com. 